and atebrin. This effect is maximal during the administration of the drug, when large doses are reached. There seems to be no residual and no delayed effects.

(2) A residual and a delayed increase brought about by the repeated administration of antigenic substances as in the case of T.A.B. vaccine or as occurs with repeated attacks of malaria and the resultant liberation of malarial proteins. This second mechanism seems to be more specific, but is distinct from the specific globulin antibody response which is also obtained in these cases. The residual effect obtained in the case of this resistance factor was maximal one week after the cessation of immunization, whereas the antibody response demonstrated by the zone of flocculation in the serial cephalin flocculation curve was maximal two weeks after the cessation of immunization.

The delayed effect comes into action whenever the immunized host is infected with the same agent.

A more detailed account is in the press.

I wish to thank Prof. E. W. Dennis, head of the Bepartment of Bacteriology and Parasitology of the American University of Beirut, for his valuable advice.

${ }^{1}$ Makari, J. G., J. Trop. Med. and Hyg., 49, 113 (1946).

*Hanger, F. M., Trans. Assoc. Amer. Phys., 53, 148 (1938).

3 Kabat, E. A., Hanger, F. M., Moore, D. H., and Landow, H., J. Clin. Invest., 22, 563 (1943).

-Moore, D. B., Pierson, P. S., Hanger, F. M., and Moore, D. H., J. Clin Invest., 24, 292 (1945).

sCohn, E. J., Oneley, J. L., Strong, L. E., Armstrong, S. H., and Hughes, W. L., Jr., J. Clin. Invest.. 28, 417 (1944).

- Dennis, E. W., and Makari, J. G., in the press.

¿Makari, J. G., J. Trop. Med. and Hyg. 49, 70 (1946).

\section{INTERSTELLAR MATTER}

$\mathrm{T}$ HE George Darwin Lecture entitled "Some Phenomena connected with Interstellar Matter" was delivered by Prof. J. H. Oort before the Royal Astronomical Society on May 10, 1946, and the lecture has now been published in full (Mon. Not. Roy. Astro. Soc., 106, 3 ; 1946). Prof. Oort deals more with a survey of the problems presented by interstellar matter than with an account of our present knowledge on the subject. The uneven distribution of interstellar matter is the first characteristic that strikes the investigator, and this applies to interstellar gas as well as to obscuring clouds. On the average, the light from a star 1,000 parsecs distant traverses five clouds, and it can now be accepted that the dark clouds contain proportionate concentration of gas. It has been shown that, on the average, the intensities of interstellar lines increase proportionally with the distances of the stars in all galactic longitudes, the only explanation of which is that these lines are mainly formed in clouds having 'peculiar' velocities averaging 15 to $20 \mathrm{~km}$./ $/ \mathrm{sec}$. in the radial component.

The very uneven distribution of obscuring matter is noticeable even in the galactic plane, and two extended systems of dark clouds are found in our neighbourhood, one in the Taurus-Auriga-Perseus region, and the other in Ophiuchus and Scorpius. Both systems cover regions of about 500 square degrees and show an average absorption of mag. $0 \cdot 8$, although they are comparatively close to us-about 100-150 parsecs. In contrast to the strong absorption in these clouds, there are very faint Cepheids in Cygnus the apparent magnitudes of which are scarcely affected by absorption, although their distances are between 10,000 and 20,000 parsecs. As the average diameters of the interstellar clouds are of the order 100 parsecs and their peculiar velocities $20 \mathrm{~km}$./sec., a cloud will encounter another in about $10^{7}$ years, and some remarkable effects will result from such an encounter. Incidentally, it might be expected that the individual cloud systems and their relative motions would disappear in a time short in comparison with the age of the galactic system; but as this has not occurred there must be some mechanism causing reformation and motion. Independent data suggest that this is taking place, and the six-colour photometry carried out by Stebbins and Whitford, as well as other evidence, show that the dependence of interstellar scattering upon wave-length is the same for all stars, no variation occurring whether observations are made through dense clouds or through a thin medium. This implies that particles within dense clouds have the same radii as outside particles ; and if it is assumed that these particles grow by condensation from the interstellar gas, the only interpretation is that the clouds are being continually reshuffled.

Two effects will result from an encounter between clouds. First of all, the intermediate region between the clouds will be compressed, and if the relative velocity of an encounter is $30 \mathrm{~km}$. $/ \mathrm{sec}$, the density and temperature in this region will be three times their value in the clouds. Hydrogen will be ionized by collisions and the gas will become luminous, the luminosity being most conspicuous when the interface between the clouds is in the line of sight from the earth. This may be the explanation of the bright rings often observed near the dark nebulæ when these are bordered by luminous nebulæ, and the luminescence of nebulæ like the extensive nebulosities near Zeta Orionis or the North American nebula may be caused by this collisional mechanism rather than by radiation from the stars.

Another effect is the evaporation of the solid particles of the two colliding clouds when the particles of one cloud hit other particles of comparable size. The probability of such evaporation cannot be ignored, and it may play an important part in the realm of interstellar material. This leads to the problem of the origin and growth of these solid interstellar particles, of which the radii are $1,000 \mathrm{~A}$. A full discussion of this last and very important question would be impossible in the limited space, but briefly it can be said that an order of magnitude of $10^{-24}$ gm./c.c. for the density should preserve a balance between condensation and evaporation.

Among other astronomical problems in which interstellar material plays an important part reference may be made to the significance of interstellar material in the extragalactic nebulæ. There is no trace of it in the elliptic nebulæ; but it is very prominent in the spiral nebulæ, especially in the case of objects seen on edge. If the dark matter were comparable in mass with all the stars in a nebula, it might lead to instability, and spiral arms would be formed. The absence of such material in the elliptic nebulæ would offer a possible explanation of their stability; but it is admitted that such suggestions are still merely speculative.

The problem of the resistance of interstellar gas to the expanding shell thrown off by a nova is considered, and it is shown that in the case of an ordinary nova expanding in an interstellar medium of average density the deceleration should be observable within half a century. For supernovæ the deceleration would become visible only after several thousand years. 